\title{
The Evaluation of a Health-Based Education and Behavioral Change Retreat on Quality of Life, Physical Activity and Psychosocial Health in Adult Cancer Survivors: An Exploratory Analysis
}

\author{
Iris Lesser ${ }^{1}$ (D) Julie-Anne Fritz ${ }^{2} \cdot$ Lisa Belanger ${ }^{3}$ \\ Published online: 4 February 2020 \\ (C) American Association for Cancer Education 2020
}

\begin{abstract}
Knights Cabin Cancer retreats may represent an ideal opportunity and environment to fill the gap in health education and behavioral change. It is unknown, however, whether the program improves quality of life, physical activity, and psychosocial health. Cancer survivors who enrolled in a retreat completed online questionnaires prior to and 3 months post to assess health-related quality of life, physical activity, and psychosocial variables; perceived stress, depression, and self-esteem. Paired $t$ tests were utilized to compare changes pre and 3 months post the retreat. The majority of the 51 cancer survivors were female with a mean age of 53. The mental component summary of health-related quality of life $(p<0.016)$ and physical activity score $(p=0.048)$ was significantly improved at follow-up, while the physical component summary of health-related quality of life was not $(p=0.139)$. In addition, participants showed an improvement in psychosocial variables of self-esteem $(p=0.016)$ and depression $(p=0.016)$ after 3 months but did not show a significant improvement in perceived stress $(p=0.054)$. Improvements were seen following a Knights Cabin Cancer retreat in mental health-related quality of life and physical activity as well as some psychosocial variables. Further research is needed to evaluate the individual program components and the sustainability of the program in the community.
\end{abstract}

Keywords Cancer survivorship · Cancer · Oncology $\cdot$ Physical activity $\cdot$ Health behavior $\cdot$ Retreat $\cdot$ Social support · Quality of life

\section{Introduction}

Improvements in medical detection and intervention [1], alongside an aging population, have resulted in a growing number of cancer survivors. Cancer survivorship is now viewed as a distinct phase in the cancer treatment spectrum with unique biomedical and psychosocial effects [2] which may affect daily functioning and quality of life [3]. Survivorship research places an emphasis on optimizing physiological, psychosocial, and functional outcomes for cancer survivors and their families [4] such as the well-documented treatment-related side effects; pain, fatigue, depression,

Iris Lesser

Iris.lesser@ufv.ca

1 Department of Kinesiology, University of the Fraser Valley, 45190 Caen Avenue, Chilliwack, BC V2R 0N3, Canada

2 Department of Science, Mount Royal University, Calgary, AB, Canada

3 Haskayne School of Business, University of Calgary, Calgary, Alberta, Canada anxiety, sleep disturbance, and reductions in aerobic capacity; and deleterious alterations in body composition $[5,6]$.

Health behaviors including greater engagement in physical activity are associated with a lower risk of diseases such as cardiovascular disease [7] and lower all cause and cancerspecific mortality [8]. This may be due to the overwhelming evidence showing that exercise in cancer survivors improves health-related fitness and quality of life [9] in addition to having a positive effect on symptoms of cancer treatment such as fatigue [10], depression [11], and sleep quality [12]. This has led to the development of physical activity guidelines specific to cancer survivors [13].

Although cancer survivors are primed for health behavior change following cancer diagnosis $[14,15]$, few cancer survivors are meeting lifestyle guidelines including physical activity and nutrition [16] due to lack of the knowledge and skills to return to positive health behaviors [17]. Healthcare professionals believe that lifestyle advice should be tailored to the individuals and should be focused on small and achievable changes, however, report feeling that they were not the "right" person to provide advice and were lacking in time and resources [18]. The need for individualized, community-based programming designed for cancer survivors, specifically 
regarding exercise programming [9], is an unfulfilled need recognized by the broader medical community [19]. Satisfying this need would provide survivors and their supporters the necessary resources and support to address some of the challenges they face post-diagnosis [20].

In 2014, Knights Cabin Cancer retreats were created as a community-based program to support cancer survivors and their supporters in their transition from cancer patient to living a life of wellness post-cancer diagnosis (Lesser et al., 2019 [21]). Survivors and their supporters can attend a weekend long health education and behavior retreat to improve the likelihood of change and maintenance in health behavior in a supportive community of other people with cancer. During the weekend, attendees can participate in guided hikes, yoga, classes on nutrition, physical activity, stress, sleep management techniques, and facilitated workshops on behavior change and habit formation. All programming is evidence based, taught by experts in the area, and based on the theory of behavior change. Retreats are an opportunity for survivors and their supporters to take the time to rest, reflect, and refocus on their health while providing the ability to connect with other survivors to increase their social support.

Knights Cabin Cancer retreats may represent an ideal opportunity and environment to fill the gap in health education and behavioral change. It is unknown, however, whether the program improves quality of life, physical activity, and psychosocial health. We therefore utilized a single group pre- and post-test study design that aimed to evaluate attendance at a Knights Cabin Cancer retreat on health-related quality of life, physical activity, and psychosocial health 3 months postretreat in cancer survivors. We hypothesized that the educational workshops, social support, and goal-setting workshop would improve quality of life, physical activity, and the associated psychosocial domains. The aim of this study is to evaluate Knights Cabin Cancer retreats on the quality of life, physical activity, and psychosocial health of participants at a 3month follow-up. This evaluation will improve Knights Cabin programming and provide future direction for Knights Cabin research.

\section{Materials and Methods}

\section{Study Population}

Participants learned of Knights Cabin Cancer retreats through word of mouth, social media, and website postings. If they were interested in participating, they contacted Knights Cabin. The potential participants were asked to get their treating physicians (oncologists or family physician) to complete a medical clearance form for physical activity (PARMED-X). The physician provides a summary of medical history, two separate blood pressures, limitations to exercise (if any) and required restrictions of exercise, or if exercise must be medically supervised by a Certified Exercise Physiologist.

If there were any questions about conditions or medications, the physician was contacted. This occurred once due to an unclear statement about a previous heart condition. The information was clarified, and the participant was cleared for unsupervised exercise. All cancer types are invited to participate in the retreat. Cancer survivors who are on immunosuppressants or who are not medically stable are excluded from participating in retreats.

\section{Knights Cabin Cancer Retreats}

Cancer survivors were encouraged to bring a supporter to the retreat in order to provide encouragement and continued assistance in achieving health behavior change as well as to help supporters in reducing compassion fatigue and improving their own health. The multidimensional focus of Knights Cabin programming is based around the health behavior components that are rooted in evidence regarding their impact on cancer survivor health in either a physical or psychosocial domain in addition to counseling on adherence to the behavior. The retreat begins with an introduction to behavior change using the theory of planned behavior due to its extensive research with cancer survivors [9]. Over the course of a weekend retreat, cancer survivors and their supporters are engaged in education on physical activity and nutrition, sleep hygiene, mindfulness workshops, stress management, and participate in activities such as yoga and hiking. All activities are done as a group with survivors and their supporters following a detailed schedule that factors in time for both educational and participatory events as well as leisure time, time blocks for educational components, and participatory events average $90 \mathrm{~min}$ (Table 1). All education provided is based on current evidence-based guidelines in the field of oncology and presented by volunteers with expertise in the area such as yoga instructors, certified exercise physiologists, dieticians, and sleep experts. At the end of the retreat, cancer survivors engage in a goal-setting workshop focusing on health habit change based on influencing different constructs of the theory of planned behavior such as social support or physical activity enjoyment. Individuals primarily chose to plan their health behavior changes around physical activity due to the emphasis placed on the importance of physical activity in cancer survivorship.

\section{Questionnaires}

Knights Cabin Cancer participants were directed to complete an online survey before and 3 months after attending a Knights Cabin Cancer retreat. Reminders for follow up survey 
Table 1 Example of Knights Cabin Cancer retreat program scheduling

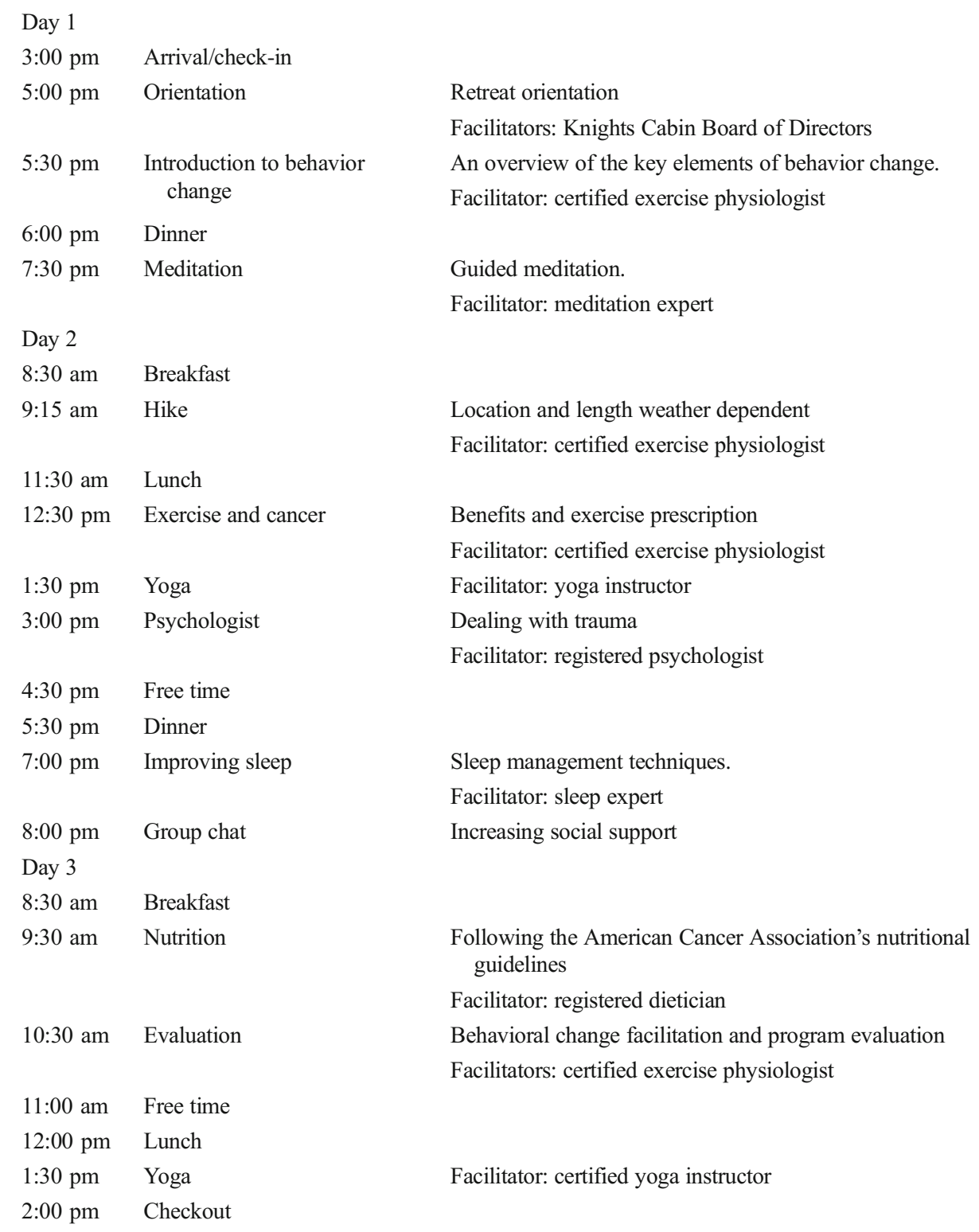

Day 1

$\begin{array}{cl}\text { 3:00 pm } & \text { Arrival/check-in } \\ \text { 5:00 pm } & \text { Orientation } \\ 5: 30 \mathrm{pm} & \begin{array}{l}\text { Introduction to behavior } \\ \text { change }\end{array} \\ & \text { Dinner } \\ 6: 00 \mathrm{pm} & \text { Meditation }\end{array}$

Day 2

8:30 am Breakfast

9:15 am Hike

11:30 am Lunch

12:30 pm Exercise and cancer

1:30 pm Yoga

3:00 pm Psychologist

4:30 pm Free time

5:30 pm Dinner

7:00 pm Improving sleep Sleep management techniques.

Facilitator: sleep expert

8:00 pm Group chat Increasing social support

Day 3

8:30 am Breakfast

9:30 am Nutrition

10:30 am Evaluation

11:00 am Free time

12:00 pm Lunch

$1: 30 \mathrm{pm} \quad$ Yoga

2:00 pm Checkout

Retreat orientation

Facilitators: Knights Cabin Board of Directors

An overview of the key elements of behavior change.

Facilitator: certified exercise physiologist

Guided meditation.

Facilitator: meditation expert

Location and length weather dependent

Facilitator: certified exercise physiologist

Benefits and exercise prescription

Facilitator: certified exercise physiologist

Facilitator: yoga instructor

Dealing with trauma

Facilitator: registered psychologist

Following the American Cancer Association's nutritional guidelines

Facilitator: registered dietician

Behavioral change facilitation and program evaluation

Facilitators: certified exercise physiologist

Facilitator: certified yoga instructor

completion were done via email prompt with a direct link to the online survey.

Demographic, medical characteristics, and cancer history were collected by self-report and included current age, gender, marital status, education, employment status, height, weight, comorbidities, body mass index (calculated by height and weight), cancer type, stage of cancer, and treatment (chemotherapy, surgery, radiation). Participants self-reported their smoking status as never smoked, having quit smoking in the last 6 months, or being a regular smoker.

Physical activity was assessed using the Godin LeisureTime Exercise Questionnaire [22]. This questionnaire was used to calculate average weekly frequency and duration of physical activity with three separate intensity categories: light (minimal effort, no perspiration), moderate (not exhausting, light perspiration), and vigorous (heart beats rapidly, sweating) over the past month. An evaluation of the Godin LeisureTime Exercise Questionnaire found a 1-month test-retest reliability of $0.62[23,24]$ and concurrent validity coefficient of 0.32 when measured against accelerometry data [24].

Health-related quality of life was assessed by the Medical Outcomes Study 36-Item Short Form (SF-36) survey [25]. The SF36 contains eight subscales that can be summed and weighted to provide a physical component summary score and a mental component summary score. Higher scores indicate better functioning. The validity and reliability of the SF-36 has been established in cancer survivors [26] revealing Cronbach's $\alpha$ coefficient for all subscales ranging from 0.60 to 0.85 , test-retest scores from 0.81 to 0.93 , and validity coefficients of $\geq 0.61$ for all subscales. 
Three scales were used to measure the psychosocial variables: perceived stress was assessed by the Perceived Stress Scale [27] which has a Cronbach's $\alpha$ of 0.87 and a test-retest reliability score of 0.86 [28]. Self-esteem was assessed by the Rosenberg Self-Esteem Scale [29] which has a Cronbach's $\alpha$ and test-retest of 0.88 and a construct validity of 0.46 to 0.71 [30]. Depression was measured by the Center for Epidemiologic Studies-Depression (CES-D) scale [31]. In an evaluation with a cancer population, the CES-D had a Cronbach's $\alpha$ of 0.89 and a test-retest score of 0.57 . The correlations between the CES-D and other depressive assessments were between 0.65 and 0.77 [32].

\section{Statistical Analysis}

Baseline data are presented as mean and standard deviation for continuous data and number and percentage for categorical data. A paired $t$ test was used to assess change in healthrelated quality of life, physical activity, perceived stress, selfesteem, and depression at baseline and 3-month follow-up. Significance was set at $p<0.05$.

\section{Results}

One-hundred thirty-seven cancer survivors and 67 of their supporters attended one of eleven Knights Cabin Cancer retreats hosted across Canada from 2014 through 2018. Data on supporters was not collected while all cancer survivors completed pre-retreat questionnaires. Of the cancer survivors who completed a pre-retreat questionnaire, only 85 of the 137 participants completed a post-retreat questionnaire at 3 months. Due to anonymous data collection and issues with data linkage, of those 85 individuals, 51 individuals are included in this evaluation.

Of the 51 individuals for whom we have pre- and postretreat survey data, mean (SD) age was 53 (10.5). The majority of cancer survivors were female $(94.2 \%)$, married or common law (64.7\%), completed university/college or higher (70.6\%), and were not currently working $(45.1 \%)$ (Table 2).

Cancer survivors self-reported height and weight with an average body mass index of 27.6 (4.6) and the majority being in the overweight or obese category (58.8\%). Most survivors underwent treatment in the form of radiation, surgery, and/or chemotherapy, and many were still receiving treatment at the time of the retreat $(45.1 \%)$. The majority of cancer survivors reported 1 or more comorbidities (53\%) with arthritis being the highest reported comorbidity (33.3\%) (Table 3).

Results of the health-related quality of life questionnaire are depicted by domains of mental and physical component summaries as well as their independent categories. The mental component summary of health-related quality of life (SF-36) was significantly improved at follow-up $(p<0.016)$, while the
Table 2 Demographic characteristics of participants $(n=51)$

\begin{tabular}{ll}
\hline Demographic variables & $N(\%)$ \\
\hline Age mean, SD(53,10.5) & \\
$30-39$ & $6(11.8 \%)$ \\
$40-49$ years & $8(15.7 \%)$ \\
$50-59$ years & $20(39.2 \%)$ \\
$>60$ years & $17(33.3 \%)$ \\
Sex & \\
Male & $3(5.9 \%)$ \\
Female & $48(94.2 \%)$ \\
Marital status & \\
Married/common law & $33(64.7 \%)$ \\
Not married/common law & $18(35.3 \%)$ \\
Education & \\
Completed university/college or higher & $36(70.6 \%)$ \\
Some university/college or lower & $15(29.4 \%)$ \\
Employment Status & \\
Employed full/part-time & $23(45.1 \%)$ \\
Not employed & $28(54.9 \%)$ \\
\hline
\end{tabular}

physical component summary was not significantly improved $(p=0.139)$. When the independent categories of healthrelated quality of life were analyzed, there was an improvement in the pain, general health, vitality, and emotional domains of health-related quality of life $(p<0.05)$ (Table 4$)$.

Participants reported an improvement in physical activity with an average score of 77 (66.5) prior to attending the retreat and an average score of 101 (14.6) 3 months post-attending a retreat $(p=0.048)$.

In addition, participants showed an improvement in their self-esteem $(p=0.016)$ and depression $(p=0.016))$ after 3month follow-up compared to before attending a Knights Cabin Cancer retreat but did not show a significant improvement in perceived stress $(p=0.054)$ (Table 4$)$.

\section{Discussion}

Cancer survivors who participated in a Knight's Cabin Cancer retreat showed an improvement in health-related quality of life in the SF-36 mental component score as well as the domains of pain, general health, vitality, and emotional health. Cancer survivors also showed an increase in physical activity and self-esteem and a reduction in depression (depression scores were only indicative of mild depression prior to the retreat). This suggests that Knights Cabin Cancer retreats are successful in improving physical activity and can have a positive impact on elements of cancer survivor health-related quality of life and psychosocial health which aligns with our hypothesis. 
Table 3 Medical characteristics of population $(n=51)$

\begin{tabular}{|c|c|}
\hline Medical variables & $N(\%)$ \\
\hline \multicolumn{2}{|c|}{ Body mass index (mean, SD) 27.6, 4.60} \\
\hline Healthy & $16(31.4 \%)$ \\
\hline Overweight & $13(25.5 \%)$ \\
\hline Obese & $17(33.3 \%)$ \\
\hline \multicolumn{2}{|l|}{ Number of comorbidities } \\
\hline None & $19(37.3 \%)$ \\
\hline 1 & $16(31.4 \%)$ \\
\hline 2 & $6(11.8 \%)$ \\
\hline$>3$ & $5(9.8 \%)$ \\
\hline \multicolumn{2}{|c|}{ Most common comorbidities } \\
\hline High blood pressure & $10(19.6 \%)$ \\
\hline High cholesterol & $7(13.7 \%)$ \\
\hline Arthritis & $17(33.3 \%)$ \\
\hline \multicolumn{2}{|l|}{ Smoking status } \\
\hline Never smoked & $33(64.7 \%)$ \\
\hline Ex-smoker & $14(27.5 \%)$ \\
\hline Current smoker & $2(3.9 \%)$ \\
\hline \multicolumn{2}{|c|}{ Stage of cancer at diagnosis } \\
\hline 1 & $12(23.5 \%)$ \\
\hline 2 & $15(29.4 \%)$ \\
\hline 3 & $13(25.5 \%)$ \\
\hline 4 & $2(3.9 \%)$ \\
\hline \multicolumn{2}{|l|}{ Type of cancer } \\
\hline Breast & $38(74.5 \%)$ \\
\hline Other & $10(19.6 \%)$ \\
\hline \multicolumn{2}{|l|}{ Treatment received } \\
\hline \multicolumn{2}{|l|}{ Surgery } \\
\hline No & $25(49.0 \%)$ \\
\hline Yes & $15(29.4 \%)$ \\
\hline \multicolumn{2}{|l|}{ Chemotherapy } \\
\hline No & $20(39.2 \%)$ \\
\hline Yes & $28(54.9 \%)$ \\
\hline \multicolumn{2}{|l|}{ Radiation Therapy } \\
\hline No & $4(7.8 \%)$ \\
\hline Yes & $47(92.2 \%)$ \\
\hline \multicolumn{2}{|l|}{ Current treatment status } \\
\hline Completed & $11(21.6 \%)$ \\
\hline Receiving treatment & $23(45.1 \%)$ \\
\hline
\end{tabular}

These results are consistent with other behavioral lifestyle interventions that improve physical activity behavior among cancer survivors which have shown improved health outcomes [33, 34]. To our knowledge, Knights Cabin offers a lifestyle approach that is unique due to its retreat format that allows for built-in social support while incorporating broader health behaviors. The improvement in physical activity reported after a Knights Cabin Cancer retreat suggests an increased interest, and short-term adherence, to apply knowledge regarding physical activity engagement. This is in line with the knowledge that cancer survivors are interested and able to participate in physical activity [35]. It is worth noting that prior to the retreat, the average physical activity score was 66 and considered to be physically active although there was a large standard deviation. A Cochrane review noted that cancer survivors who have greater physical activity participation also showed higher health-related quality of life and reduced anxiety, fatigue, pain, and sleep disturbance [36]. Although not the objective of this paper, we also noted a significant correlation ( $p=0.024)$ between the general domain of healthrelated quality of life and physical activity score.

In addition to engaging participants in physical activity education, counseling, and practice, Knights Cabin Cancer retreats focus on a holistic approach to health behavior. This includes a focus on the practice of mindfulness, stress management, sleep health, and behavioral modification. An approach that broadly focuses on stress and coping is essential given the high amount of psychosocial distress that is reported among cancer survivors [37]. Retreat participants report significant reductions in depression (although baseline values were not clinically significant) and improvements in perceived stress, however, are not at a significant level $(p=0.054)$, suggesting that this broad approach may improve health behavior with a positive impact on psychosocial health. Broad approaches such as mindfulness have previously been shown to improve a range of symptoms among breast cancer survivors that includes the anxiety associated with fear of recurrence [38].

Our exploratory analysis of Knights Cabin Cancer retreats has important strengths and limitations. One of the strengths of the study is that, to the best of our knowledge, this is the first multidimensional community-based health behavior retreat for adult cancer survivors in Canada that has assessed physical and psychosocial health impact. We believe there is a greater likelihood of engagement in this program due to it occurring over the course of a weekend allowing for working adults with many commitments to engage in self-care. The effectiveness of unsupervised, home-based exercise programming compared with supervised, facility-based exercise programming has been assessed. Compared with exercising independently, exercise in facilities utilizing group formatting prompted greater adherence, potentially due to the social factors that patients are exposed to [39]. The retreat format is cost-effective compared with supervised exercise training while allowing for the necessary social factors to improve adherence.

This exploratory analysis suggests that Knights Cabin Cancer retreats may have a positive impact on the quality of life, physical activity, and psychosocial health of cancer survivors. However, given that retreats are a one-time occurrence, future research is necessary to determine whether regular follow up after a retreat is more effective at facilitating and sustaining health behavior changes. For instance, while we saw a significant improvement in physical activity, it was modest and may suggest challenges in adhering to physical 
Table 4 Pre- and post-scores of health-related quality of life, perceived stress score, selfesteem, depression, and physical activity in cancer survivors who attended a Knights Cabin Cancer retreat $(n=51)$

\begin{tabular}{llll}
\hline & Pre Mean (SD) & Post Mean (SD) & $p$ value \\
\hline $\begin{array}{l}\text { SF-36 health-related quality of life } \\
\text { Physical component summary }\end{array}$ & $40.09(8.07)$ & $41.45(7.92)$ & 0.139 \\
$\quad$ Mental component summary & $38.06(10.87)$ & $41.53(10.92)$ & 0.016 \\
Quality of life subscales & & & \\
Physical functioning & $71.60(24.65)$ & $75.32(21.58)$ & 0.104 \\
Role physical & $28.92(42.24)$ & $32.35(43.96)$ & 0.530 \\
Bodily pain & $57.92(19.07)$ & $64.75(21.80)$ & 0.012 \\
General health & $56.80(22.56)$ & $61.71(21.98)$ & 0.025 \\
Vitality & $43.19(23.00)$ & $53.24(24.80)$ & 0.001 \\
Social functioning & $69.61(25.65)$ & $72.79(24.20)$ & 0.364 \\
Role emotional & $37.25(43.02)$ & $50.33(45.89)$ & 0.040 \\
Mental health & $68.71(18.76)$ & $73.18(20.10)$ & 0.102 \\
Perceived stress score & $24.55(8.49)$ & $22.16(9.83)$ & 0.054 \\
Self-esteem score & $28.57(7.65)$ & $31.12(5.97)$ & 0.016 \\
Depression score & $8.74(5.63)$ & $6.98(6.26)$ & 0.016 \\
Physical activity score & $77.90(66.5)$ & $101.4(15.56)$ & 0.048 \\
\hline
\end{tabular}

SF-36 health-related quality of life. Higher score represents a more favorable health state

Perceived stress score. Higher score represents a higher perceived rate of stress

Self-esteem score. Higher scores indicate higher self-esteem

Depression score. Higher scores indicate greater severity of depression. Less than 4 is minimal or none, 5-9 mild, and $10-14$ moderate

Physical activity score. A leisure score index of greater than 24 is considered active inclusive of light, moderate, and vigorous activity with higher weightings with intensity activity programming post-retreat. Future research aims to engage participants throughout the follow-up period to increase the likelihood of successful behavior maintenance. In addition, more information needs to be gathered regarding cancer survivor healthcare needs posttreatment and whether Knights Cabin programming meets the gaps in healthcare provisions. It is also worth noting that the majority of cancer survivors who attend Knights Cabin programming are women with breast cancer, and there may be different outcomes in other survivor populations. It has been noted that while women with breast cancer make up $22 \%$ of the survivor population, they represent $60 \%$ of the participants in randomized trials [40].

\section{Study Limitations}

The most significant limitations of our study is likely the selfreport of physical activity and medical data and our small sample size due to low response rate and missing data that led to an underpowered sample for the research question. The low response rate and missing data is a weakness of this analysis as we were only able to make pre-post comparisons on the 51 participants who completed follow-up questionnaires. One of the reasons for low response rate may be due to not providing sufficient email reminders regarding questionnaire completion. Three email reminders were added in at later retreats which dramatically improved completion rates. In addition, confidentiality was achieved by having individuals use initials only when completing baseline and follow-up questionnaires; however, due to inconsistency in the initials, there was further loss of data due to an inability to match data. Additionally, it should be noted that there were no corrections for multiple comparisons in analysis due to the small sample size. Other potential limitations of this pilot work are a lack of data to provide evidence regarding other health changes that may have occurred between attending a Knights Cabin Cancer retreat and the time of completing a follow-up questionnaire. For instance, participant feedback suggests that there may have been some additional health challenges, such as cancer reoccurrence, which occurred during this 3-month follow-up that could have affected program impact; however, this was not captured. Lastly, there is no comparative control group, and therefore, we are unable to know whether the changes observed would have occurred independent of attending a retreat.

\section{Clinical Implications}

Knights Cabin Cancer retreats have the potential to improve health-related quality of life and physical activity and improve psychosocial health among cancer survivors. Given the call for innovative approaches bridging clinical and community 
resources in survivorship care [41], Knights Cabin Cancer retreats represents a potential solution to this complex problem. However, it is necessary to assess the ability of this type of programming to be sustainable and provide equal access to care within a diverse population of cancer survivors. In addition, it is imperative that a further understanding of the clinical impact of the individual program aspects to be evaluate to isolate their independent contribution to health outcomes.

Data Availability Statement The data that support the findings of this study are available on request from the corresponding author. The data are not publicly available due to privacy or ethical restrictions.

\section{Compliance with Ethical Standards}

Conflict of Interest Dr. Lisa Belanger is the founder of Knights Cabin Cancer Retreats and was responsible for its evidence based design. Dr. Belanger was independent from all data analyses done by Dr. Iris Lesser and Dr. Julie-Anne Fritz and has no financial investment or benefit from Knights Cabin Cancer Retreats. The authors declare that they have no conflict of interest.

Ethical Approval All procedures performed in studies involving human participants were in accordance with the ethical standards of the institutional and/or national research committee and with the 1964 Helsinki declaration and its later amendments or comparable ethical standards. Ethics approval was obtained from the University of the Fraser Valley Human Research Ethics board (1085 K-18).

Informed Consent Data was originally collected by Knights Cabin Cancer Retreats with participant knowledge with a specific statement that survey data would be used for program evaluation purposes. University of the Fraser Valley was asked as an independent investigator to complete a program evaluation at this time a letter of information was sent to all participants explaining the further use of data for academic publication.

\section{References}

1. Burke S, Wurz A, Bradshaw A, Saunders S, West MA, Brunet J (2017) Physical activity and quality of life in Cancer survivors: a meta-synthesis of qualitative research. Cancers 9(5):53

2. Institute of Medicine (2006) From Cancer Patient to Cancer Survivor: Lost in Transition. National Academies Press, Washington, DC

3. Wu HS, Harden JK (2014) Symptom burden and quality of life in survivorship: a review of the literature. Cancer Nurs 38:E39-E54

4. Aziz NM (2007) Cancer survivorship research: state of knowledge, challenges and opportunities. Acta Oncol 46(4):417-432

5. Kenefick AL (2006) Patterns of symptom distress in older women after surgical treatment for breast cancer. Oncol Nurs Forum 33: 327-335

6. Fallowfield L, Jenkins V (2015) Psychosocial/Survivorship Issues in Breast Cancer: Are We Doing Better? J Natl Cancer Inst 107(1):335

7. Keats MR, Cui Y, Grandy SA, Parker L (2017) Cardiovascular disease and physical activity in adult cancer survivors: a nested, retrospective study from the Atlantic PATH cohort. J Cancer Surviv 11:264-273
8. Lahart IM, Metsios GS, Nevill AM, Carmichael AR (2015) Physical activity, risk of death and recurrence in breast cancer survivors: a systematic review and meta-analysis of epidemiological studies. Acta Oncol 54(5):635-654

9. Courneya KS (2017) Exercise guidelines for cancer survivors: are fitness and quality-of-life benefits enough to change practice? Curr Oncol 24(1):8-9

10. Tian L, Lu HJ, Lin L, Hu Y (2016) Effects of aerobic exercise on cancer-related fatigue: a meta-analysis of randomized controlled trials. Support Care Cancer 24:969-983

11. Craft LL, Vaniterson EH, Helenowski IB, Rademaker AW, Courneya KS (2012) Exercise effects on depressive symptoms in cancer survivors: a systematic review and meta-analysis. Cancer Epidemiol Biomark Prev 21:3-19

12. Courneya KS, Segal RJ, Mackey JR, Gelmon K, Friedenreich CM, Yasui Y, Reid RD, Jespersen D, Cook D, Proulx C, Trinh L, Dolan LB, Wooding E, Forbes CC, McKenzie D (2014) Effects of exercise dose and type on sleep quality in breast cancer patients receiving chemotherapy: a multicenter randomized trial. Breast Cancer Res Treat 144:361-369

13. Rock CL, Doyle C, Demark-Wahnefried W, Meyerhardt J, Courneya KS et al (2012) Nutrition and physical activity guidelines for cancer survivors. CA Cancer J Clin 62:242-274

14. Demark-Wahnefried W, Aziz NM, Rowland JH, Pinto BM (2005) Riding the crest of the teachable moment: promoting long-term health after the diagnosis of cancer. J Clin Oncol 23(24):5814-5830

15. Vijayvergia N, Denlinger CS (2015) Lifestyle factors in cancer survivorship: where we are and where we are header. J Pers Med 5(3):243-263

16. Williams K, Steptoe A, Wardle J (2013) Is a cancer diagnosis a trigger for health behaviour change? Findings from a prospective, population-based study. Br J Cancer 108:2407-2412

17. Irwin ML, Crumley D, McTiernan A, Bernstein L, Bamugartner R, Gilliland FD, Kriska A, Ballard-Barbash R (2003) Physical activity levels before and after a diagnosis of breast carcinoma: the health, eating, activity and lifestyle (HEAL) study. Cancer 97(7):1746-1757

18. Koutoukidis DA, Lopes S, Fisher A, Williams K, Croker H, Beeken RJ (2018) Lifestyle advice to cancer survivors: a qualitative study on the perspectives of health professionals. BMJ Open, 2018; 8(3):e020313

19. Halpern MT, Viswanathan M, Evans TS, Birken SA, Basch E, Mayer DK (2015) Models of cancer survivorship care: overview and summary of current evidence. J Oncol Pract 11:e19-e27

20. Hewitt M, Rowland JH, Yancik R (2003) Cancer survivors in the United States: age, health and disability. J Gerontol A Biol Sci Med Sci 58:82-91

21. Lesser I, McGowan E, Belanger L (2019) Letter to the Editor: The development of knights cabin cancer retreats: a community program to engage cancer survivors' proactive health behaviors. Appl. Cancer Res. 39, 14 (2019)

22. Godin G, Jobin J, Bouillon J (1986) Assessment of leisure time exercise behaviour by self report: a concurrent validity study. Can J Public Health 77(5):359-362

23. Okun M, Karoly P, Lutz R (2002) Clarifying the contribution of subjective norm to predicting leisure-time exercise. Am J Health Behav 26(4):296-305

24. Goldberg DP, Rickels K, Downing R, Hesbacher P (1976) A comparison of two psychiatric screening tests. Br J Psychol 129:61-67

25. Ware J, Snow KK, Kosisnki M, Gandek B (1993) SF-36 health survey: manual and interpretation guide. The Health Institute, New England Medical Center, Boston

26. Pinar R (2004) Reliability and construct validity pf the SF 36 in Turkish cancer patients. Qual Life Res 14(1):259-264

27. Cohen S, Kamarck T, Mermelstein R (1983) A global measure of stress. J Health Soc Behav 24(4):385-396

28. Reis RS, Hino A, Anez CR (2010) Perceived stress scale: reliability and validity study in Brazil. J Health Psychol 15(1):107-114 
29. Rosenburg M (1965) Society and the adolescent self-image. Princeton University Press, Princeton, p 326

30. Robins R, Hendin HM, Trzesniewski KH (2001) Measuring global self- esteem: construct validation of single-item measure and the Rosenburg self esteem scale. Personal Soc Psychol Bull 27:151

31. Andersen EM, Malmgren JA, Carter WB, Patrick DL (1994) Screening for depression in well older adults: evaluation of a short form of the CES-D (Center for Epidemiologic Studies Depression Scale). Am J Prev Med 10(2):77-84

32. Hann D, Winter K, Jacobsen P (1999) Measurement of depressive symptoms in cancer patients: evaluation of the Centre for epidemiological studies depression (CES-D). J Psychosom Res 46(5):437-443

33. Stacey FG, James EL, Chapman K, Courneya KS, Lubans DR (2015) A systematic review and meta-analysis of social cognitive theory-based physical activity and/or nutrition behavior change interventions for cancer survivors. J Cancer Surviv 9:305-338

34. Rogers LQ, Hopkins-Price P, Vicari S, Pamenter R, Courneya KS, Markwell S, Verhulst S, Hoelzer K, Naritoku C, Jones L, Dunnington G, Lanzotti V, Wynstra J, Shah L, Edson B, Graff A, Lowy M (2009) A randomized trial to increase physical activity in breast cancer survivors. Med Sci Sports Exerc 41(4):935-946

35. Wong JM, McAuley E, Trinh L (2018) Physical activity programming and counseling preferences among cancer survivors: a systematic review. Int J Behav Nutr Phys Act 15:48
36. Mishra SI, Schere RW, Giegle PM, Berlanstein DR, Topaloglu O, Gotay CC, Snyder C (2012) Exercise interventions on health related quality of life for cancer survivors. Cochrane Database Syst. Rev. 2012 Aug 12; (8)

37. Ganz PA, Guadagnoli E, Landrum MB, Lash TL, Rakowski W, Silliman RA (2003) Breast cancer in older women: quality of life and psychosocial adjustment in the 15 months after diagnosis. $\mathrm{J}$ Clin Oncol 21:4027-4033

38. Lengacher CA, Reich RR, Paterson CL, Ramesar S, Park JY, Alinat C, Johnson-Mallard V, Moscoso M, Budhrani-Shani P et al (2016) Examination of broad symptom intervention resulting from mindfulness-based stress reduction in breast cancer survivors: a randomized controlled trial. J Clin Oncol 2016 Aug 20; 34(24): 2827-34.

39. Jackson C, Dowd AJ, Capozzi LC, Brisel W, Lau HY, Culos Reed SN (2018) A turning point: head and neck cancer patients; exercise preferences and barriers before and after participations in an exercise intervention. Eur J Cancer Care (Engl) 27(2):e12826

40. American Cancer Society. Cancer Treatment \& Survivorship Facts \& Figures 2016-2017. Atlanta: American Cancer Society; 2016

41. White MC, Hayes NS, Richardson LC (2015) Public health's future role in cancer survivorship. Am J Prev Med 49(6, 5):S550-S553

Publisher's Note Springer Nature remains neutral with regard to jurisdictional claims in published maps and institutional affiliations. 